\title{
A Relativistic Electron-Positron Outflow from a Tepid Fireball
}

\author{
Katsuaki Asano ${ }^{1}$ And Fumio Takahara ${ }^{2}$ \\ asano@phys.titech.ac.jp, takahara@vega.ess.sci.osaka-u.ac.jp
}

\begin{abstract}
Through detailed numerical simulations, we demonstrate that relativistic outflows (Lorentz factor $\Gamma \sim 7$ ) of electron-positron pairs can be produced by radiative acceleration even when the flow starts from a nearly pair equilibrium state at subrelativistic temperatures. Contrary to the expectation that pairs annihilate during an expansion stage for such low temperatures, we find that most pairs can survive for the situations obtained in our previous work. This is because in the outflow-generating region the dynamical timescale is short enough even though the fireball is optically thick to scattering. Several problems that should be solved to apply to actual active galactic nucleus jets are discussed.
\end{abstract}

Subject headings: plasmas — relativity — galaxies: jets

\section{Introduction}

One of the most challenging problems in astrophysics is the acceleration mechanism of relativistic jets from active galactic nuclei (AGNs) and Galactic black hole candidates. The bulk Lorentz factor of these jets is above 10 and the kinetic power is almost comparable to the Eddington luminosity. Although various jet models have been proposed, we do not yet have a satisfactory solution. Recent remarkable progress in magnetohydrodynamical (MHD) simulations includes Poynting-dominated jet formation by a rapidly rotating black hole with an accretion disk (e.g. Mckinney 2006; Hawley \& Krolik 2006). Along the spin axis a centrifugal funnel filled with magnetic field is formed. The magnetic field in the funnel is considered to be amplified by the differential rotation of the accretion disk and the framedragging effect of the black hole. However, in the simulation of Mckinney (2006), the total

\footnotetext{
${ }^{1}$ Interactive Research Center of Science, Graduate School of Science, Tokyo Institute of Technology, 2-12-1 Ookayama, Meguro-ku, Tokyo 152-8550, Japan

${ }^{2}$ Department of Earth and Space Science, Graduate School of Science, Osaka University, Toyonaka 5600043, Japan
} 
jet luminosity within the funnel is only $0.2 \%$ of the rest-mass energy accretion rate. While continuous developments in numerical studies are expected to probe MHD acceleration, other types of acceleration mechanisms are worth considering.

One of the alternative acceleration mechanisms is the thermal expansion of optically thick fireballs. If the matter content of jets is electron-positron pairs, thermal radiation pressure from accretion disks may be sufficient to accelerate the jets. The original model of the fireball (Rees \& Mészáros 1992) relevant to gamma-ray bursts (GRBs) assumes that its initial stage is radiation-dominated plasmas that are in complete thermal equilibrium at high temperatures comparable to the electron rest-mass energy $m_{\mathrm{e}} c^{2}$. However, the characteristic size of AGNs is too large to achieve complete thermal equilibrium. The novel model proposed to overcome this problem is electron-positron pair outflow from a "Wien fireball" (Iwamoto \& Takahara 2002, 2004), which is a photon-dominated, optically thick plasma, but the densities of photons and pairs are less than those in the complete thermal equilibrium fireballs. Pairs coupled with photons by scattering are thermally accelerated, expending the internal energy of the fireball. As is seen in the original model of the fireball, the Lorentz factor $\Gamma=1 / \sqrt{1-\beta^{2}}$ increases with the radius $R$, and the temperature drops as $T \propto R^{-1}$. Iwamoto \& Takahara (2002, 2004) showed that a sufficient amount of electron-positron pairs survive as a relativistic outflow, if the temperature of the fireball at the photosphere is relativistic $\left(\gtrsim m_{\mathrm{e}} c^{2}\right)$.

When the temperature is relativistic, all the cross-sections of pair creation, annihilation and Compton scattering are of the same order. Therefore, a balance between pair creation and annihilation is realized, and the number densities of pairs and photons are of the same order as long as photons and pairs are coupled with each other. On the other hand, the condition to make the pair-annihilation timescale shorter than the dynamical timescale,

$$
n_{+} \sigma_{\mathrm{T}} \frac{R}{\Gamma \beta}>\frac{3}{8}\left[1+\frac{2 \theta^{2}}{\ln (1.12 \theta+1.3)}\right],
$$

is almost the same as the condition of being optically thick to scattering, where $n_{+}, \sigma_{\mathrm{T}}$, and $\theta=T / m_{\mathrm{e}} c^{2}$ are the positron density in the comoving frame, Thomson cross section, and the normalized temperature, respectively. This implies that the number of pairs is almost conserved outside the photosphere, where photons and pairs are decoupled. Therefore, a relativistic temperature at the photosphere is required to obtain a sufficient amount of pairs avoiding annihilation.

The temperature of fireballs is determined by microscopic processes in their formation sites, presumably accretion disks, such as radiative cooling, Compton scattering, and $\gamma-\gamma$ pair production (see e.g. Svensson 1984). Assuming that pairs are confined with protons, several authors (see e.g. Kusunose 1987; Björnsson \& Svensson 1992, and references there 
in) investigated pair equilibrium plasmas. However, a series of studies of pair equilibrium plasmas implies that the equilibrium temperature is too low for the Wien fireball model. The plasma temperature typically becomes $\theta \sim 0.1$ for plasmas of size $\sim 10^{14} \mathrm{~cm}$ and luminosity $\gtrsim 10^{45} \mathrm{erg} \mathrm{s}^{-1}$, while the Wien fireball model requires a super-Eddington luminosity $\gtrsim 10^{47}$

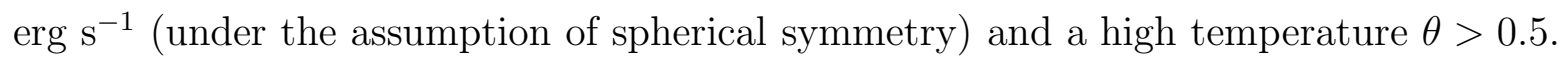

The previous studies on pair equilibrium plasmas have not taken into account the dynamical effects of outflowing electron-positron pairs on the plasma temperature. It is not trivial to estimate the motional (or advective) effects of fireballs on radiative transfer, cooling processes, pair density and temperature. In our previous paper (Asano \& Takahara 2007, hereafter AT07), we simultaneously solved the dynamics of outflowing pairs, microphysics, and radiative transfer to obtain the internal structure of fireballs. Even in this simulation, we obtained only $\theta \sim 0.2$ at $R \sim$ several times $10^{14} \mathrm{~cm}$ for the luminosity $L=10^{47} \mathrm{erg}$ $\mathrm{s}^{-1}$, though a considerable amount of pairs outflow with a mildly relativistic velocity. In the simulations of AT07, the acceleration of outflows is not finished within the simulation scale. We pessimistically supposed that a rapid extinction of pairs inside the photosphere may occur, since the obtained temperature is so low.

However, the detailed situations in AT07 are different from the simplified inner boundary conditions of the outflow in the simulations of Iwamoto \& Takahara (2004). The pairphoton ratio at the outer boundary of the simulation region is smaller than that of the Wien equilibrium plasma by a factor of $\sim 2$. The photon spectrum obtained numerically is also different from the simple Wien spectrum. It may be valuable to simulate subsequent evolution of the outflows outside such a tepid fireball obtained in the simulations in AT07.

In this Letter, we demonstrate that the tepid fireball obtained by the simulations of AT07 can be accelerated to a relativistic velocity conserving the pair number, even though the fireball temperature is not high enough. In \$2, we describe our simulation method, and show our results in 93 , 94 is devoted to discussion.

\section{Method}

\subsection{Energy-Release Region}

We numerically obtain spherically symmetric, steady solutions of radiation and pair outflows. As the inner boundary condition, we employ the numerical values obtained at the outer boundary in the simulations of AT07. Namely, the simulations of AT07 provide us with the formation stage of the fireball. First of all, let us review the physical situations in 
AT07. In AT07, the proton number density is assumed to be

$$
n_{p}(R)=n_{0} \exp \left[-\left(R / R_{0}\right)^{2}\right]
$$

where $R_{0}$ and $n_{0}$ are constant. In this region the plasma was assumed to be heated, which mimics energy release via viscous heating. The heating rate of the plasma was proportional to $n_{p}$. The plasmas were divided into three fluids: proton $(p)$, background electron $(e)$, and pair $\left(e^{ \pm}\right)$fluids. The background electrons and protons were assumed to be static; the gravitational force of the central black hole may arrest the background plasma. Although the validity of this multifluid approximation is not always assured, we had adopted this approximation for simplicity. For the microphysics, Coulomb scattering, Compton scattering, bremsstrahlung, and electron-positron pair annihilation and creation were taken into account. The frictional forces and heating of the pair plasma due to Coulomb scattering with background fluids were calculated using numerical results from Asano et al. (2007). The effect of the frictional force was less important than the radiative force. The spherical symmetry in the geometry and the mildly relativistic outflow yield beamed photon distribution. It is a well-known effect that too fast flows are decelerated even by a beamed radiation field (Compton drag). The flow velocity was self-regulated by the beamed photon field. Radiative transfer was solved with the Monte Carlo method. Taking into account Compton scattering and pair creation, the evolutions of energy and direction along their trajectories are fully solved for each photon. The energy range of photons $x \equiv \varepsilon / m_{\mathrm{e}} c^{2}$ was from $10^{-5}$ to $10^{2}$, and the outer boundary was set at $R=R_{\text {out }} \equiv 2 R_{0}$. For the total heating rate $L=10^{47}$ erg s${ }^{-1}$, AT07 obtained mildly relativistic $(U \equiv \Gamma \beta \sim 1)$ outflows. The ratios of the total luminosity to the mass ejection rate, $\eta \equiv L / 4 \pi R_{\text {out }}^{2} n_{ \pm}\left(R_{\text {out }}\right) U\left(R_{\text {out }}\right) m_{\mathrm{e}} c^{3}$, are $\sim 30$, but the temperatures are relatively low $(\theta \sim 0.2)$.

\subsection{Outer Region}

Using $U, n_{ \pm}, \theta$ and beamed photon field at the outer boundary in AT07 as the inner boundary conditions, we simulate the behavior outside the energy-release region (the simulation region of AT07). The detailed method of the simulations is the same as that in AT07. However, we assume that protons do not exist anymore, so we switch off heating and the Coulomb friction due to background protons and electrons. Thus, we take into account only Compton scattering, bremsstrahlung, and electron-positron pair annihilation and creation for the outer region. We solve hydrodynamics and radiative transfer only for pair fluids. 


\section{Results}

In AT07 two sets of parameters were adopted: $R_{0}=10^{14} \mathrm{~cm}$ with $n_{0}=10^{10} \mathrm{~cm}^{-3}$, and $R_{0}=3 \times 10^{14} \mathrm{~cm}$ with $n_{0}=\frac{1}{3} \times 10^{10} \mathrm{~cm}^{-3}$. These values are characteristic of accretion plasmas in AGNs. In both cases, the Thomson scattering depth due to the $e$-fluid $\tau_{\mathrm{p}} \sim n_{0} R_{0} \sigma_{\mathrm{T}} \sim 0.7$. The basic features of the obtained outflows are similar in these two cases. The resultant density of the pair plasma overwhelms the density of the background plasma so that $n_{0}$ may not be a critical parameter. Although the results for the latter parameter set were explained in detail in AT07, we show the results employing the former compact case as an energy-release region in Figs. 1 and 2. The outflow is accelerated mainly by a radiative force. While the photosphere is located at $\sim 10^{15} \mathrm{~cm}$, the acceleration turns out to continue even outside the photosphere. Even though the fraction of photons interacting with the plasma is small, the influence of radiative interaction outside the photosphere cannot be neglected because of the copious amount of photons. The final Lorentz factor becomes $\sim 7$.

Fig. 2 represents the behavior of the electron temperature and exhibits our rather artificial inner boundary conditions in the simulation. Within $R=2 R_{0}$ (the boundary between the energy-release region and the outer region), the heating from the background protons makes electron temperature increase with radial distance. The sudden shutdown of the heating at $R=2 R_{0}$ results in the discrete change of temperature gradient. Outside the energy-release region the temperature decreases monotonically, but the temperature drop begins to slow down around the photosphere. Outside the photosphere, the simple analytical formulae for fireballs $\left(U \propto R\right.$ and $\left.T \propto R^{-1}\right)$ are no longer applicable.

If the plasma is in the Wien equilibrium, a drastic extinction of pairs should occur for such a low temperature. We also plot the positron number flux $R^{2} n_{+} U$ in Fig. 1. The dashed line (result from AT07) shows that most of the outflowing material is provided in the outer part of the energy-release region. Outside the energy-release region, even though the temperature decreases from $\theta \sim 0.2$ to 0.03 at the photosphere, the number of pairs is

almost conserved. At $R=2 R_{0}$, the pair-annihilation timescale is estimated as $\sim 2 \times 10^{3} \mathrm{~s}$ in the comoving frame, while the timescale of density drop $\left(n_{+}\right.$becomes half in this timescale) due to plasma expansion is $\sim 10^{3} \mathrm{~s}$. Thus, the two timescales are already comparable even though $2 R_{0}$ is inside the photosphere. Since the pair-annihilation timescale $\propto n_{+}^{-1}$, we can neglect the pair annihilation outside $2 R_{0}$. Photons and pairs are not in the Wien equilibrium, though the plasma is optically thick to scattering. This is because the pair plasma keeps on being heated during the initial acceleration, and is smoothly evacuated outward at a mildly relativistic velocity. If the temperature drop begins further inside, lower electron temperature may lead to rapid annihilation of pairs.

A problem is that the energy outflow rate of the pair plasma is only $\sim 1 / 60$ of the 


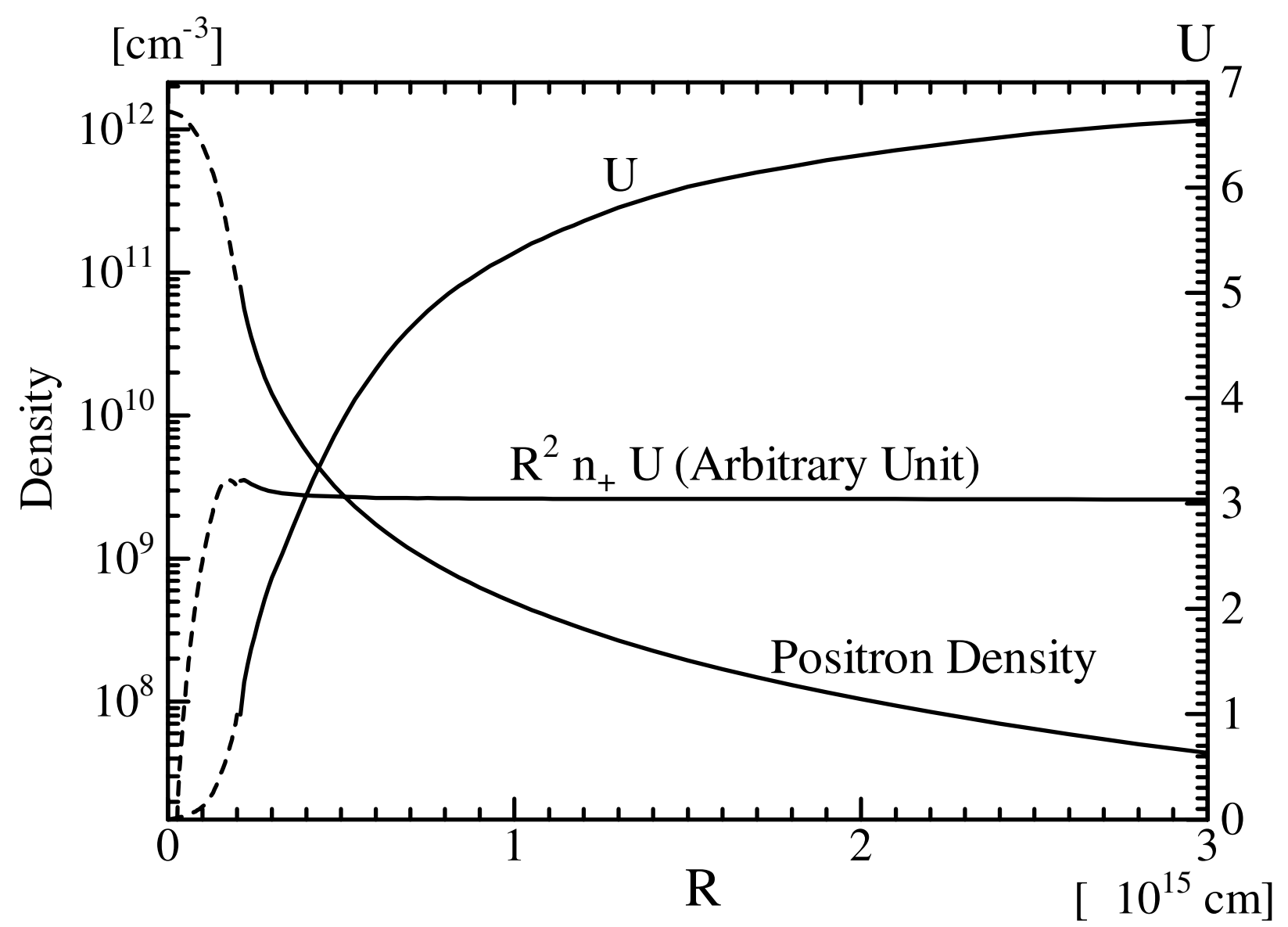

Fig. 1.- Distributions of the positron density in the comoving frame $n_{+}$(left axis), 4-velocity $U$ (right axis), and $R^{2} n_{+} U$ (arbitrary units, see the left axis for reference). The dashed lines are the results from AT07.

total luminosity $L$. The rest of the energy escapes as photons from the boundary. In Fig. 3. we plot the energy spectrum of the photons escaping from the outer boundary of this simulation $L_{\gamma}(x) d x\left(x \equiv \varepsilon / m_{\mathrm{e}} c^{2}\right)$. The spectrum has a broader feature than that in the Wien spectrum. There are huge amounts of soft photons $\left(x<10^{-2}\right)$ in comparison with the thermal spectrum. As shown in Fig. 4, the photon field is highly beamed. As for X-ray photons $\left(x \sim 10^{-2}\right)$, the off-axis flux is diluted by a factor of $\sim 100$ compared with the on-axis flux.

We also simulate an outflow for the broader profile of protons: $R_{0}=3 \times 10^{14} \mathrm{~cm}$ with $n_{0}=\frac{1}{3} \times 10^{10} \mathrm{~cm}^{-3}$. The qualitative results are the same as the results described above, but the final Lorentz factor $\Gamma \sim 5$. Given the luminosity $L$, the radius of the photosphere may not change drastically. Therefore, considering the behavior $\Gamma \propto R$, the larger initial 


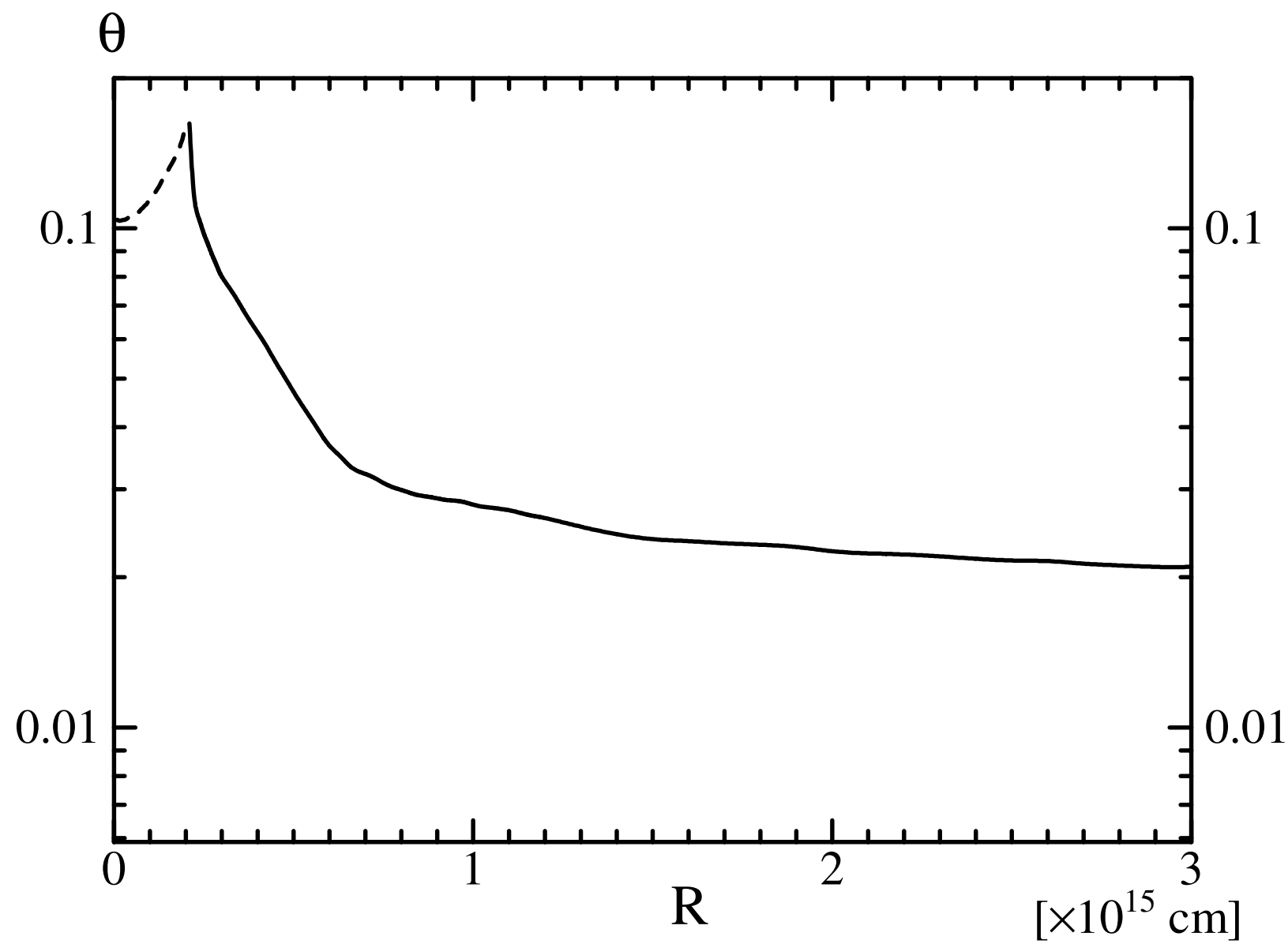

Fig. 2.- Temperature of the pair plasma. The dashed line is the result from AT07.

radius (namely the radius at $U \sim 1$ ) results in a smaller Lorentz factor. On the other hand, the energy outflow rate of the pair plasma is $14 \%$ of the total luminosity $L$, which is larger than the former case. While a more compact energy release is preferable to increase the final Lorentz factor, conversely the efficiency of the energy conversion into pair outflow becomes larger for a more capacious source.

\section{Discussion}

Our simulation shows that an energy release of $10^{47} \mathrm{erg} \mathrm{s}^{-1}$ within a size of $\sim 10^{14}$ $\mathrm{cm}$ produces a pair outflow of $\Gamma \sim 7$. Even though the fireball temperature is tepid, rapid annihilation of pairs is avoided. If the entire system we have considered is moving with a Lorentz factor $\Gamma_{\mathrm{s}} \geq 1.06\left(\beta_{\mathrm{s}} \geq 0.34\right)$, the Lorentz factor of the pair plasma seems to be larger 


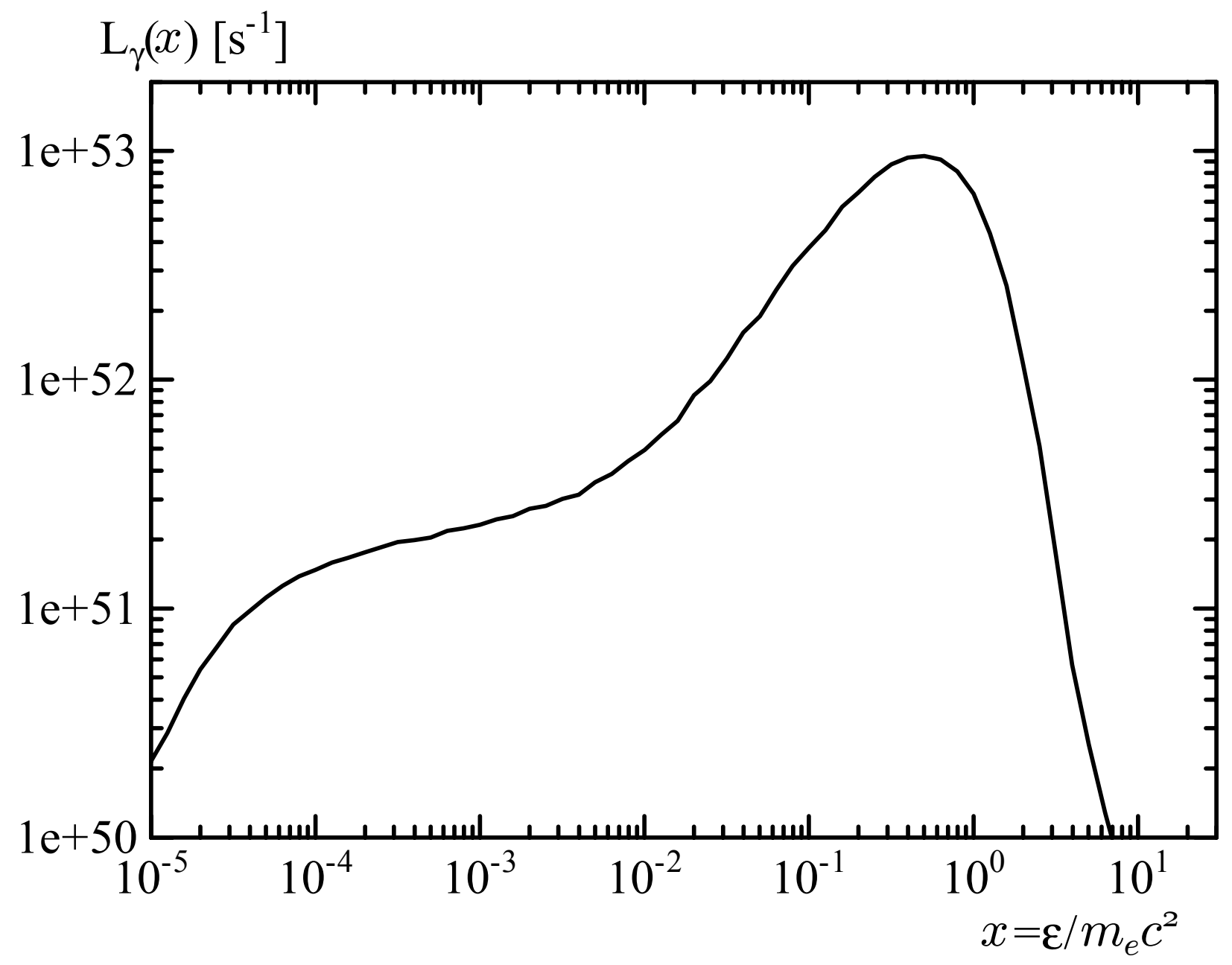

Fig. 3.- Energy spectrum of the photons escaping from the outer boundary.

than 10. Such a nonrelativistic outflow of protons may be easily achieved, as various MHD simulations show. Therefore, similar simulations to ours including the outflow of protons are valuable, while we have assumed that the background protons are static.

The reason why a rapid annihilation of pairs does not occur may be a slight difference in pair density from the prediction of the Wien equilibrium. This difference comes from the finite size of the energy-release region. In the energy-release region the outflowing material keeps on being heated and is evacuated with a mildly relativistic velocity. The rapid expansion makes the pair plasma escape smoothly from the optically thick region, avoiding pair annihilation. For such a marginal optical depth, the Wien equilibrium is no longer a reasonable approximation. The pair densities obtained numerically are suitable for avoiding pair annihilation and keeping the plasma optically thick to scattering, which are opposing 
requirements in general.

However, resultant densities are too low to convert the radiation energy into the kinetic energy of the pair plasma efficiently. The efficiency in our simulation is only $1 / 60$ of the total luminosity. The results may depend on the profiles of the plasma heating rate. Simulations for other types of profiles of the plasma heating rate are worth studying. We do not simulate

for $L>10^{47} \mathrm{erg} \mathrm{s}^{-1}$, because the higher pair density requires high computational cost, while the temperature tends to decrease with $L$. This is another challenge for us, though there is a possibility of rapid extinction of pairs due to the lower temperature.

Of course, the most idealized method to efficiently produce relativistic outflows is to create fireballs with relativistic temperatures. Relativistic temperatures for optically thick plasmas assure a higher density of pairs, which is preferable for both the energy efficiency and the final Lorentz factor. However, as AT07 showed for steady solutions, even if the effects of mildly relativistic motion are taken into account, the temperature of fireballs is close to that of static pair equilibrium plasmas obtained by past studies. It is valuable to seek a much faster solution $(U>1)$ than AT07. As is usually seen in solutions of the accretion disk, by varying the boundary conditions we may obtain another type of solution with high temperature and high outward advection. However, it is difficult to make $U>1$ within the energy-release region, because the effect of Compton drag may be crucial in such a compact region. The photon field is produced from the plasma itself so that the photon distribution is not sufficiently beamed in the inner region, where the plasma is produced. We expect plasma acceleration only in the outer region, where the photon distribution is highly beamed.

Another possibility is nonsteady solutions of outflows. We should note that the timescale of radiative transfer is much longer than the dynamical timescale. The lifetime of accretion plasma may not be long enough to achieve the steady-state radiation field obtained here and in AT07. As in the original idea of the Wien fireball model, an instantaneous heating may produce a runaway production of relativistic pairs because of slow annihilation in a hot plasma. Therefore, time-dependent simulations should be examined in a future work.

We appreciate the anonymous referee for his/her helpful advice. This work is supported in part by Scientific Research Grants (F.T. 18540239 and 20540231) from the Ministry of Education, Culture, Sports, Science and Technology of Japan.

\section{REFERENCES}

Asano, K., Iwamoto, S., \& Takahara, F. 2007, ApJS, 168, 268 
Asano, K., \& Takahara, F. 2007, ApJ, 655, 762 (AT07)

Björnsson, G., \& Svensson, R. 1992, ApJ, 394, 500

Hawley, J. F., \& Krolik, J. H. 2006, ApJ, 641, 103

Iwamoto, S., \& Takahara, F. 2002, ApJ, 565, 163

Iwamoto, S., \& Takahara, F. 2004, ApJ, 601, 78

Kusunose, M. 1987, ApJ, 321, 186

Mckinney, J. C. 2006, MNRAS, 368, 1561

Rees, M. J., \& Mészáros, P. 1992, MNRAS, 258, 41P

Svensson, R. 1984, MNRAS, 209, 175 


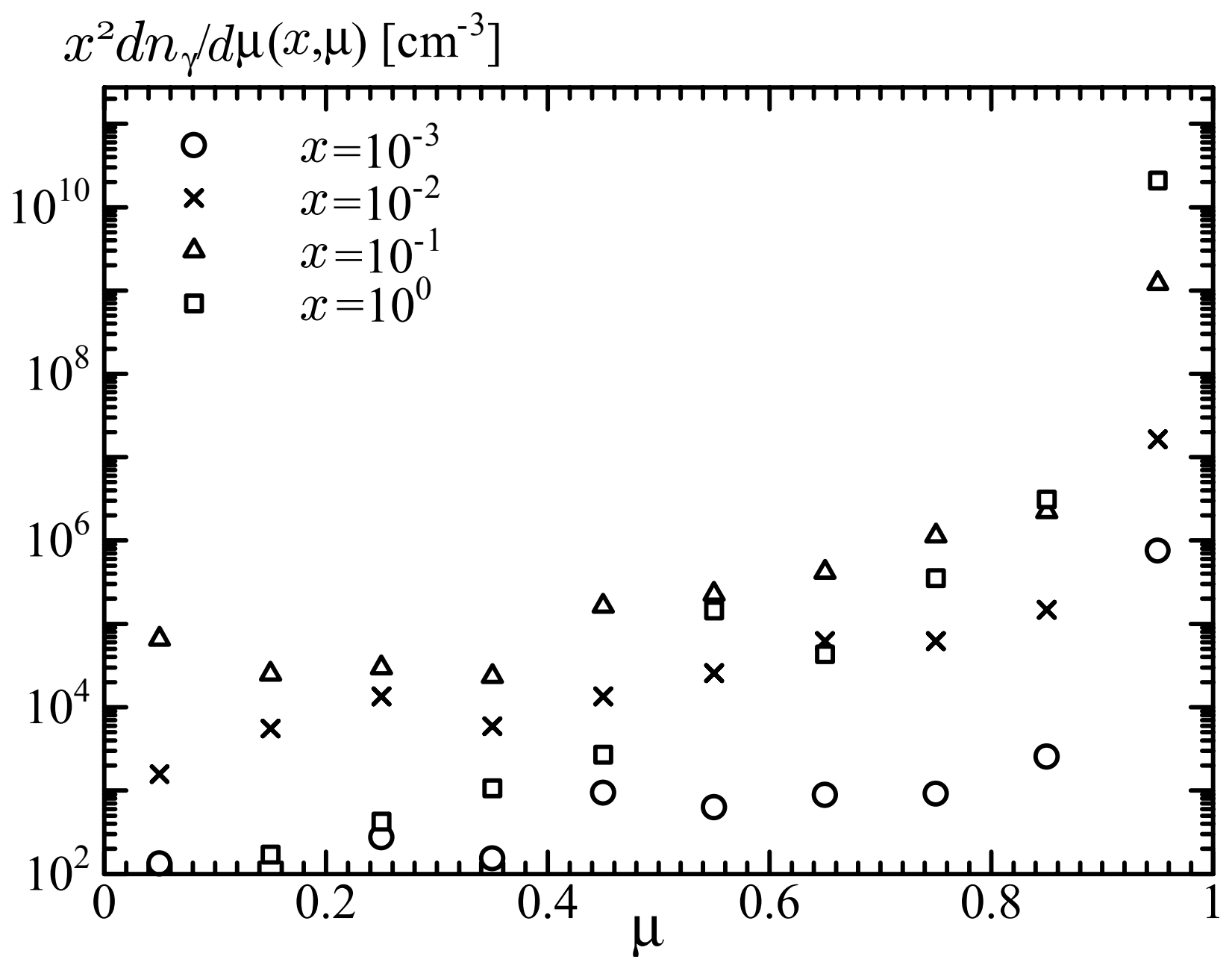

Fig. 4.- Angular distribution of photons at the outer boundary, where $\mu$ is the cosine between the direction of the photon and the radial direction. The results are plotted from binned data with $\Delta \mu=0.1$ after following $1.2 \times 10^{9}$ paths of photons. 